\title{
Somewhere Together: Location, Parsimony and Multilocation
}

\section{Roberto Loss}

robertoloss.com

Forthcoming in Erkenntnis

$\underline{\text { Penultimate draft: } \text { please refer to the published version }}$

\begin{abstract}
Most of the theories of location on the market appear to be ideologically parsimonious at least in the sense that they take as primitive just one locative notion and define all the other locative notions in terms of it. Recently, however, the possibility of some exotic metaphysical scenarios involving gunky mixtures and extended simple regions of space has been argued to pose a significant threat to parsimonious theories of locations. The aim of this paper is to show that a theory taking as primitive a notion of plural pervasive location and allowing for irreducibly plural locative facts can account for all the putatively problematic scenarios for parsimonious theories of location. Furthermore, I will also argue that the notion of plural pervasive location is compatible with the possibility of multilocation.
\end{abstract}

\section{Introduction}

Almost all the theories of location that have been proposed in the literature so far appear to be theoretically parsimonious, at least in the sense that they take just one locative notion to be primitive and indefinable. ${ }^{1}$ Consider, as a way of example, the two theories that Parsons (2007) labels 'S@' and 'S@o'. The first takes as primitive the notion of exact location (the 'shadow' of an object in substantival space; see Parsons 2007: 203) and defines the notions of weak and pervasive location as follows (' $x \leq y$ ' stands for ' $x$ is part of $y$ ' and ' $x \circ y$ ' for ' $x$ overlaps with $y$ '):

(1) $\quad x$ is weakly located at $r=_{\mathrm{df}} x$ is exactly located at a region overlapping $r$ $x @_{o} r={ }_{d f} \exists s(x @ s \wedge s \circ r)$

(2) $\quad x$ is pervasively located at $r=_{\mathrm{df}} x$ is exactly located at a superregion of $r$ $x @_{>} r={ }_{d f} \exists s(x @ s \wedge r \leq s)$

The second takes as primitive the notion of weak location (a region that is not 'completely free' of the relevant object; see Parsons 2007: 203) and defines the notions of pervasive and exact location as follows:

(3) $x$ is pervasively located at $r=_{\mathrm{df}} x$ is weakly located at every region overlapping $r$ $x @_{>} r={ }_{d f} \forall s\left(r \circ s \rightarrow x @_{o} s\right)$

\footnotetext{
${ }^{1}$ See, among others, Casati and Varzi (1999), Parsons (2007), Gilmore (2018) and Kleinschmidt (2014). As Kleinschmidt (2016: 188, fn 5) notices, possible exceptions to this claim are represented by the theories defended by Fine (2006) and Simons (2014), who posit different relations for objects located in space and those located in time.
} 
(4) $\quad x$ is exactly located at $r=_{\mathrm{df}}$ for every region $s, s$ overlaps $r$ if and only if $x$ is weakly located at $s^{2}$

$x @ r={ }_{d f} \forall s\left(r \circ s \leftrightarrow x @_{o} s\right)^{3}$

Recently, however, some authors have argued that certain seemingly conceivable metaphysical scenarios appear to be intractable by parsimonious theories of location. Consider, for instance, the case of Nolan's (2006) 'Stoic gunk'. Imagine pouring equal parts of some gunky water and gunky wine in a glass enclosing a gunky region of space and have the water and the wine mix through and through. After the blending, every region inside the glass contains both water and wine, and yet there is no region inside the glass containing only water or only wine. As Leonard (2014) has shown, neither $S @$ and $S @{ }_{o}$ appear to be capable of accounting for this scenario. In this case, in fact, the following three claims seem to be all true:

(5) Neither the water nor the wine have an exact location

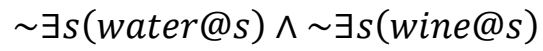

(6) Neither the water nor the wine pervade any region of space $\sim \exists s\left(\right.$ water@ $\left.@_{>} s\right) \wedge \sim \exists s\left(\right.$ wine@ $\left.@_{>} s\right)$

(7) Both the water and the wine are weakly located at every subregion of the region $r$ at which their blend is exactly located ${ }^{4}$

$\forall s\left(s \leq r \rightarrow\right.$ water@o $s \wedge$ wine $\left.@_{o} s\right)$

However, (5)-(7) are jointly inconsistent under the assumption of either $S @$ or $\mathrm{@}_{o}$, thus seemingly forcing friends of Stoic Gunk to take both the notions of weak and exact location to be primitive (Leonard 2014: 312-3). Similarly, Kleinschmidt (2016) has argued that, under the assumption of some seemingly plausible recombination principle, the possibility of extended simple regions of space is incompatible with parsimonious theories of location. Suppose, for instance, that there is an extended simple region of space having the size and shape of the Statue of Liberty. Imagine, furthermore, someone 'dropping' an almond in that region of space. Intuitively, the almond is not big enough to be able to fill the extended simple region. It follows that the almond cannot be said to be exactly located at that region. Therefore, Parsons's system S@ cannot even get off the ground in this case. On the other hand, the almond clearly appears to be weakly located at the extended simple region.

\footnotetext{
${ }^{2}$ Parsons (2007: 204-5).

${ }^{3}$ In addition, the notion of entire location can be defined in $\mathrm{S} @$ and $\mathrm{S} @ \mathrm{O}$, respectively, as follows:

(ENT-@) $x$ is entirely located at $r=_{\mathrm{df}} x$ is exactly located at a subregion of $r$ $x @<=_{d f} \exists s(x @ s \wedge s \leq r)$

(ENT-@ $\left.@_{O}\right) x$ is entirely located at $r==_{\mathrm{df}} x$ is weakly located at $r$ and all the regions at which $x$ is weakly located overlap $r$ $x @_{<} r={ }_{d f} x @_{o} r \wedge \forall s\left(x @_{o} s \rightarrow r \circ s\right)$

${ }^{4}$ See also Loss (2019: 252; 257)
} 
However, the extended simple region is also the only region at which the almond is weakly located. It follows, thus, from S@o that the almond is pervasively and exactly located at that region. The argument appears to generalize. Therefore, concludes Kleinschmidt, if the relevant recombination principle (allowing us to 'drop' the almond in the extended simple region) is accepted, it follows that parsimonious theories of location are incompatible with the possibility of extended simple regions of space.

Friends of ideologically parsimonious theories of location can choose to dispute the very possibility of the cases in question. Perhaps what all of this shows is only that things like Stoic Gunk and extended simple regions of space containing objects that are smaller than the regions themselves are either impossible or conceptually confused. ${ }^{5}$ However, this doesn't appear to be the only possible option on the table. In fact, as Loss (2019) has recently argued, the case of Nolanian Stoic Gunk (or 'gunky Spritz' as Loss calls it) can be accounted for within a theory that takes as its only primitive notion the notion of plural pervasive location and allows for irreducibly plural locative facts of the form 'the plurality of entities $x x$ collectively fill the region $r$ '. As Loss shows, not only does it seem possible to define all the standard locative notions in terms of the notion of plural pervasive location, but the resulting theory-which he labels ' $x x @_{>} S$ ' - appears to be perfectly able to account for the possibility of gunky Spritz. In the case of a gunky Spritz, in fact, what happens is that the water and the wine collectively fill the gunky glass and every region of space in the gunky glass even though there is no region of space that is filled only by the water or only by the wine (Loss 2019: 257).

Drawing on Loss (2019), the aim of this paper is to argue, first, that the possibility of Kleinschmidt's 'Almond in the Void' cases is also not a threat to ideological parsimony. As I will show, in fact, there appears to be an intuitive and plausible way to extend Loss's $x x @_{>} S$ that makes it perfectly capable of accounting for Kleinschmidt's 'Almond in the Void' cases. Second, I will also argue that, although Loss's $x x @_{>} S$ cannot account for the possibility of multilocation, there appears to be a way to interpret its axioms that (modulo a slight modification of the theory) avoids this limitation, so that the kind of ideological parsimony defended in this paper shouldn't be thought of as conditional on the impossibility of multilocation.

I will proceed as follows. In section 2 I will briefly summarize the main definitions and axioms of Loss's $x x @_{>} S$. In section 3 I will present some further axioms that can be added to $x x @_{>} S$ concerning the idea that regions of space themselves may be seen as 'filling' other regions of space and show how the resulting theory can accommodate all of Kleinschmidt's problematic scenarios. Finally, in section $4 \mathrm{I}$ will argue that it seems possible to formulate $x x @_{>} S$ by means of a notion of pervasive location ('basic filling') which allows for the possibility of multilocation. I will, thus, conclude that the possibility of exotic cases like Nolanian's Stoic Gunk or Kleinschmidt's Almond in the Void can be accepted without having to either reject the possibility of multilocation or offend the aesthetic sense of those who have a taste for desert ideological landscapes.

\footnotetext{
${ }^{5}$ For some recent discussion on extended simple regions and the possibility of Kleinschmidt's problematic cases see Goodsell, Duncan and Miller (2019: 8-10) and Eagle (2019: 166-7).
} 


\section{Plural pervasive location}

In the literature on location it is common to take the main locative notions to be one-one and to allow, thus, only one object to bear the relevant locative relation to a certain region of space (or spacetime). Instead, the theory Loss (2019) labels ' $x x @ \oint_{>} S$ ' allows the primitive notion of the theory - that is, the notion of plural pervasive location - to be manyone, thus making it possible for many objects to only collectively pervade a certain region of space. ${ }^{6}$ An intuitive gloss on the notion of plural pervasive location can be given as follows: a plurality of objects $x x$ collectively pervade or, as we can say, 'fill', a certain region $r$ just in case $r$ is not free of any of the $x x$ (so that each of the $x x$ contributes to fill $r$ ) and every subregion of $r$ is not free of all the $x x$ (so that the $x x$ do not leave out any part of $x$, so to say). Within this framework, singular pervasive location is just the limiting case of plural pervasive location. To say that a single object $x$ is pervasively located at a region $r$ is to say that $r$ is filled by the ('improper') plurality $x x$ of all the entities that are identical to $x$.

Some cases of plural pervasive location are pretty standard and can be explained in terms of singular pervasive location. For instance, the fusion $s$ of the regions $r_{1}$ and $r_{2}$ at which each of my hands is exactly located is such that my hands collectively fill it. In this case, we have that my hands, taken together, collectively fill $s$ because they singularly fill $r_{1}$ and $r_{2}$, and $s$ is the mereological sum of $r_{1}$ and $r_{2}$. However, within $x x @_{>} S$ not every case of collective pervasive location can be reduced to singular locative facts. According to $x x @_{>} S$, it is in principle possible for a plurality of objects to fill a region of space in a way that is irreducibly plural. The possibility of irreducibly plural pervasive location is the first ingredient of the present account of Kleinschmidt's Almond in the Void cases.

In what follows, ' $x x$ ', ' $y y$ ', ' $z z$ ', are plural variables, ' $x<y y$ ' stands for ' $x$ is one of the $y y$ ', and ' $f(x x)$ ' stands for 'the fusion of the $x x$ '. ${ }^{7}$ Furthermore, the variables ' $r$ ', ' $s$ ', ' $r r$ ', 'ss' are thought of as ranging only over regions and pluralities or regions (more on regions below: see section 3). The primitive notion of $x x @ @_{>} S$ is expressed by means of the multigrade two-place predicate '@ ' thought of as capable of having both singular and plural terms in its first place (so that both formulas like ' $x @_{>} r$ ' and ' $x x @_{>} r$ ' are taken to be well-formed). ${ }^{8,9}$ The derivative notions of $x x @_{>} S$ are the following:

\footnotetext{
${ }^{6}$ Hudson (2005) may have been the first one to consider the possibility of a fundamental locative location that is plural in one of its places (many thanks to an anonymous referee for this Journal). In fact, Hudson (2005) assumes that regions may be identified with pluralities of points, which appears to entail that facts concerning single objects being located at single regions may be construed as facts concerning single objects being located at pluralities of points, taken together: 'When being ontologically serious about regions, I prefer that they be identified either with pluralities of or with mereological fusions of concrete, unextended, simple points.' (Hudson 2005: 17).

${ }^{7}$ Following Loss (2019), I take an entity $x$ to be a fusion of the $y y$ just in case (i) each of the $y y$ is part of $x$ and (ii) every part of $x$ overlaps some of the $y y$.

${ }^{8}$ On multigrade predicates see Oliver and Smiley (2004).

9 The choice of taking the two-place predicate '@ , to be multigrade in its first place entails that, for every $x$ and $y y$ such that $x$ is the only entity among the $y y$ (so that the $y y$ are an 'improper' plurality of entities), the fact that $x @_{>} r$ and the fact that $y y @_{>} r$ are the same fact. Alternatively, one could take $@_{>}$to be strictly manyone. In that case the only way to express what I call here 'singular' pervasive location would be that of
} 
(8) $x$ helps fill $r$ ('partial pervasive location') $=_{\mathrm{df}} x$ is one of a plurality of entities that collectively fill $r$

$$
x @_{>}^{p} r=_{d f} \exists y y(y y @>r \wedge x \prec y y)
$$

(9) $\quad x$ is omnipresent at $r==_{\mathrm{df}} x$ helps fill every subregion of $r$

$$
x \operatorname{Pr}={ }_{d f} \forall s\left(s \leq r \rightarrow x @_{>}^{p} s\right)
$$

(10) $x$ is maximally omnipresent at $r=_{\mathrm{df}} x$ is omnipresent at $r$ and every region at which $x$ is omnipresent and that has $r$ as a part is identical to $r^{10}$ $x M r={ }_{d f} x \operatorname{Pr} \wedge \forall s((x P s \wedge r \leq s) \rightarrow r=s)$

(11) $\quad x$ is exactly located at $r==_{\mathrm{df}} x$ fills $r$ and every region that is filled by $x$ and that has $r$ as a part is identical to $r$ $x @ r={ }_{d f} x @>r \wedge \forall s\left(\left(x @_{>} s \wedge r \leq s\right) \rightarrow r=s\right)$

(12) $x$ is weakly located at $r=_{\mathrm{df}} x$ is maximally omnipresent at a region overlapping $r$ $x @_{o} r={ }_{d f} \exists s(x M s \wedge s \circ r)^{11}$

Notice that while the notion of weak location is defined in terms of maximal omnipresence (and, thus, in terms of the notion of partial pervasive location), the notion of exact location is defined in terms of singular full pervasive location. This leaves open the possibility that an object lack an exact location while possessing a weak location.

The list continues as follows:

(13) the $x x$ are all in $r=_{\mathrm{df}}$ each of the $x x$ helps fill some subregion of $r$ $x x I N r={ }_{d f} \forall x\left(x \prec x x \rightarrow \exists s\left(s \leq r \wedge x @_{>}^{p} s\right)\right)$

(14) the $x x$ cover $r=_{\mathrm{df}}$ every subregion of $r$ is such that at least one of the $x x$ helps fill it:

$$
x x \operatorname{COVr}=_{d f} \forall s\left(s \leq r \rightarrow \exists x\left(x<x x \wedge x @_{>}^{p} s\right)\right)
$$

\footnotetext{
employing a plural term standing for an improper plurality of entities. Here I choose the first option mainly for simplicity's sake.

${ }^{10} \mathrm{I}$ am assuming in this paper a classical mereology of regions of space, which guarantees the existence of the fusion of all the regions of space (for some discussion on the possibility of 'junky' or 'knuggy' space see Parsons 2007: 209-10).

${ }^{11}$ In addition, the notion of entire location can be defined as follows:

(ENT- $x x @>S$ ) $x$ is entirely located at $r==_{\mathrm{df}} x$ is maximally omnipresent at a subregion of $r$ $x @_{<} r=_{d f} \exists s(x M s \wedge s \leq r)$
} 
(15) the $x x$ are all pervasively in a plurality $s s$ of regions $=_{\mathrm{df}}$ each of the $x x$ fills at least one of the $s s$

$x x I N_{>} s s={ }_{d f} \forall x\left(x \prec x x \rightarrow \exists s\left(s \prec s s \wedge x @_{>} s\right)\right)$

(16) the $x x$ pervasively cover a plurality $s s$ of regions $=_{\mathrm{df}}$ each of the $s s$ is filled by at least one of the $x x$

$x x \operatorname{COV}_{>} s s={ }_{d f} \forall s\left(s \prec s s \rightarrow \exists x\left(x \prec x x \wedge x @_{>} s\right)\right)$

Notice that while 'all in' and 'cover' are defined in terms of objects partially pervading subregions of a certain singular region $r$, 'all pervasively in' and 'pervasively cover' are instead defined in terms of objects fully pervading regions belonging to a certain plurality of regions. This difference is important when it comes to providing the three axioms governing the notion of plural pervasive location:

Axiom 1 If for some plurality $s s$ of regions, $r$ is the fusion of the $s s$ and the $x x$ are all pervasively in the ss and pervasively cover the $s s$, then the $x x$ collectively fill $r$

$\exists s s\left(x x I N_{>} s s \wedge x x C O V_{>} s s \wedge r=f(s s)\right) \rightarrow x x @_{>} r$

Axiom 2 If the $x x$ collectively fill $r$, then the $x x$ are all in $r$ and cover $r$ $x x @>r \rightarrow(x x I N r \wedge x x C O V r)$

Axiom 3 If $x$ fills $r$, then it also fills every subregion of $r$ $x @_{>} r \rightarrow \forall s\left(s \leq r \rightarrow x @_{>} s\right)$

Axiom 1 takes care of the standard cases of collective pervasive location. Consider again the region $s$ that is the fusion of the two regions $r_{1}$ and $r_{2}$ at which my hands are exactly located. My hands are all pervasively in those regions (as each hand fills one of them) and pervasively cover them (as each region is filled by one of my hands). In this case, it follows from axiom 1 that my hands collectively fill $s$. The antecedent of axiom 1, however, is not true in every case in which a region $r$ is collectively filled by some plurality $x x$ of entities (so that the converse of axiom 1 is not a theorem of $x x @>S$ ). In fact, when a plurality of objects collectively fill a region in a way that is irreducible to singular location, some of the objects in question fail to fill by themselves any region of space (more on this below). Axiom 2 guarantees that, in that case, the objects are nevertheless all in $r$ (so that each of them contributes to fill $r$ ) and cover $r$ (so that no part of $r$ is free of all of them). Axiom 3 states, quite plausibly, that filling a region entails filling each of its subregions.

As Loss (2019) argues, Nolan's (2006) 'Stoic gunk' provides a perfect example of irreducibly plural pervasive location. In that case, in fact, we can say that although the water and the wine fail to singularly fill the glass (there is not enough of either of them), they do fill the glass together, so that every subregion of the region enclosed by the glass is filled by both the water and the wine. Given the definitions just provided it follows that, although both the water and the wine lack an exact location, they are nevertheless weakly located in the glass, which is intuitively as it should be. 
$x x @_{>} S$ validates the principle that Josh Parsons called 'Functionality':

Functionality No entity has more than one exact location ${ }^{12}$

$$
(x @ r \wedge x @ s) \rightarrow r=s
$$

(see Loss 2019: 257). Functionality excludes the possibility of multilocation. I will address the issue of multilocation below (section 5). For the time being, notice that Kleinschmidt clearly intends her main claim to apply also to theories of location endorsing Functionality (Kleinschmidt 2016: 120-21). Finally, $x x @_{>} S$ invalidates the principle Parsons called 'Exactness':

Exactness If something has a weak location, then it also has an exact location

$$
\exists r(x @ r) \rightarrow \exists r(x @ r)
$$

However, it validates a close enough principle, which Loss (2019) calls 'Precision':

Precision If something has a weak location, then it is also maximally omnipresent somewhere.

$$
\exists r\left(x @_{o} r\right) \rightarrow \exists r(x M r)
$$

\section{How to fill a region of space}

So far, we have only considered cases in which regions are filled by (one or many) objects. However, nothing seems to debar us from taking also regions of space to be capable of filling other regions. This move is not new in the literature on location. Casati and Varzi (1999: 123) define the notion of region by saying that a region is an entity that is exactly located at itself. In our case, we can instead take a region to be an entity that fills itself: ${ }^{13}$

(17) $\quad x$ is a region $=_{\mathrm{df}} x$ fills itself

$$
R x={ }_{d f} x @>x
$$

We can then add the following three axioms to our theory:

Axiom 4 If $x$ fills $y$, then $y$ is a region ${ }^{14}$

$$
x @_{>} y \rightarrow R y
$$

Axiom 5 If $x$ is a region filling $y$, then $y$ is part of $x$ $\left(R x \wedge x @_{>} y\right) \rightarrow y \leq x$

\footnotetext{
12 Parsons (2007: 205).

${ }^{13}$ Many thanks to an anonymous referee for this Journal for suggesting me to follow Casati and Varzi's (1999) approach and to (somehow) define the notion of region in locative terms.

${ }^{14}$ Casati and Varzi (1999: 121) call a similar principle (formulated by means of the notion of exact location) 'Conditional Reflexivity'.
} 
Axiom 6 If each of the $x x$ is a region, their fusion is also a region

$$
\forall y(y \prec x x \wedge R y) \rightarrow R f(x x)
$$

Axiom 4 guarantees that only regions can be filled by other entities. From (17) and axiom 3 it follows that every region fills each of its subregions. Therefore, from (17) and axioms 3 and 5 it follows that, if $x$ and $y$ are regions, then $x$ fills $y$ if and only if $y$ is part of $x$.

$$
(R x \wedge R y) \rightarrow(x @>y \leftrightarrow y \leq x)
$$

(17), axioms 3 and 4 entail that if $x$ is a region and $y$ is a part of $x$, then $y$ is a region too:

$$
(R x \wedge y \leq x) \rightarrow R y
$$

It also follows from (17), axiom 5, the anti-symmetry of parthood, and the definition of exact location given in (11) that (as in Casati and Varzi's system) every region is exactly located at itself:

(20) $R x \rightarrow x @ x^{15}$

In turn, this result can be used to show that, while the general principle of Functionality follows from axiom 1 (Loss 2019: 257), the principle that we might call 'Region Functionality' - which prohibits multilocation for regions - can be proved independently from axiom 1 and just from (11), (17), axiom 5, and the anti-symmetry of region-parthood:

Region Functionality Every region is exactly located only at itself

$$
R x \rightarrow \exists ! y(x @ y \wedge x=y)^{16}
$$

The importance of this result will become apparent when we address the issue of multilocation in section 4. In fact, the way in which entities will be allowed to be multilocated will involve a slight modification of axiom 1 . Therefore, the fact that (11), (17), axiom 5, and the anti-symmetry of region-parthood alone entail Region Functionality shows that even in the new framework regions will not be allowed to be multilocated, which appears to be a clear desideratum of any theory of location. Finally, axiom 6 guarantees that, as it is highly plausible, any fusion of regions is itself a region. There may be other

\footnotetext{
${ }^{15}$ Proof. Suppose $x$ is a region. By (17) $x$ fills itself. Suppose that $x$ fills a region $s$ and that $x$ is part of $s$. From axiom 5 we have that, since $x$ fills $s$ and $x$ is a region, then $s$ is part of $x$. By the anti-symmetry of parthood (a theorem of classical mereology; see footnote 9), it follows that $x$ is identical to $s$. We have thus (i) $x$ fills $x$ and (ii) for every region $s$, if $x$ fills $s$ and $x$ is a part of $s$, then $x$ identical to $s$. It follows from the definition of exact location given in (11) that $x$ is exactly located at $x$.

${ }^{16}$ Proof. Suppose that $x$ is a region that is exactly located at a region $y$. From (17) we have that $x$ fills itself. From the definition of exact location given in (11) we have that $x$ fills $y$. By axiom 5, $y$ is part of $x$. However, since $x$ is exactly located at $y$ it also follows from (11) that every region $s$ that $x$ fills and that has $y$ as part is identical to $y$. Therefore, $x$ is identical to $y$.
} 
constraints on regions that can be added to $x x @_{>} S .{ }^{17}$ However, axioms 4-6 appear to be perfectly sufficient for the purpose of this paper, so I will not pursue this line further here (notice that, for readability's sake, in what follows I will keep using ' $r$ ', 's', ' $r r$ ', 'ss', et cetera as variables ranging over regions and pluralities of regions, respectively).

Equipped with the definition of region given in (17) and axioms 4-6 we can finally move on to the final ingredient of my response to Kleinschmidt's Almond in the Void cases. We can proceed in two steps. Consider, first, the region $r$ at which my right thumb is exactly located. My right thumb fills $r$. Also my right hand fills $r$. Therefore, there is a plurality ss of regions such that (i) $r$ is the fusion of the $s s$ and (ii) my right hand and my right thumb, taken together, are both pervasively in the ss and pervasively cover the ss: the (improper) plurality of regions that are identical to $r$ itself. It follows, thus, from axiom 1 that $r$ is filled also by my right thumb and my right hand taken together. It may be objected in this case that, although (i) both my right hand and my right thumb contribute to fill $r$ and (ii) every part of $r$ is not free of them, we also have that (iii) both my hand and my thumb manage to fill $r$ by themselves. But this is just a case of harmless overdetermination. Notice that we are already accepting - as it is quite uncontroversial — that my hand and my thumb both singularly fill $r$. The move from a plurality of singular locative facts to a single plural locative fact appears to be unproblematic in this case.

Our second and final step consists in accepting that a region can be collectively filled by a plurality of entities comprising both objects and regions. Consider, for instance, an object $x$ that is exactly located at a region $r$. In this case it follows from our axioms that $r$ is not only filled both by $x$ and by $r$ itself singularly, but also by $x$ and $r$ taken together. Consider now Kleinschmidt's Almond in the Void case. What $x x @_{>} S$ (once extended by means of (17) and axioms 4-6) allows us to say is that that the region $r$ in which the almond has been dropped is collectively filled by $r$ and the almond taken together, even if there is no region of space that the almond fills by itself. In fact, not only is this claim perfectly compatible with our axioms, but it strikes one as highly plausible given what we said thus far about the notion of plural pervasive location. Axioms 1-6 do not require that, if an entity $x$ partially fills a certain region, then there is a region that is fully filled by $x$. In the case of Stoic gunk, for instance, both the water and the wine partially fill every subregion of the region enclosed by the glass in which they have been poured without filling by themselves any region of space. In the Almond in the Void case, the almond intuitively fails to completely fill the extended simple region: it is just not big enough. At the same time, however, it appears also very intuitive to say that the almond partially fills the extended simple region, given that the almond is indeed located within the extended region. Our theory of location allows us to express the notion of partially filling a region $r$ without appealing to the mereological structure of $r$. The almond doesn't partially fill $r$ because it

\footnotetext{
${ }^{17}$ Since $x x @_{>} S$ allows for irreducibly plural locative facts, the following principle (banning irreducibly plural locative facts when the entities that are located at regions are themselves regions) also appears to be highly plausible:

Axiom 7 If the $r r$ collectively fill $s$, then some regions $t t$ are such that the $r r$ are all pervasively in the $t t$, pervasively cover the $t t$ and $s$ is the fusion of the $t t$$$
r r @_{>} s \rightarrow \exists t t\left(r r I N_{>} t t \wedge r r C O V_{>} t t \wedge s=f(t t)\right)
$$

Notice that axiom 7 is just the converse of axiom 1 restricted to regions.
} 
fills a subregion of $r$. The almond partially fills $r$ because the almond and $r$ collectively fill $r$, while the almond fails to fill $r$ by itself.

Since in this case we can also say that every superregion $s$ of $r$ is such that $s$ is filled by $s$ and the almond taken together, what is peculiar about $r$ is that it is, mereologically speaking, the smallest region that the almond helps fill. From the definition of weak and entire location ${ }^{18}$ it also follows that, although the almond has no exact location, it is maximally omnipresent at $r$ and so it is both weakly and entirely located at $r$, which is, intuitively, precisely as it should be. Therefore, this theory of location can also easily distinguish between Kleinschmidt's Almond in the void case and the case she calls 'Almond in the Shadow', where the latter is just like the former except that the extended simple region in which the almond is dropped is of the same shape and size of the almond itself. In the Almond in the Shadow case, the almond doesn't only help fill the extended simple region, but it also manages to fill it by itself, and so (being the extended simple region the maximal region the almond fills) the almond turns out to be also exactly located at the extended simple region, contrary to what happens in the Almond in the Void case.

The present theory can also accommodate the case that Kleinschmidt (2016: 133-4) calls 'Out of Place Tiles'. Figure 1 represents four adjacent square extended simple regions composing a bigger square region. A square extended simple object of the same size and shape of each of the four small square regions lies 'at the center' of the bigger region. Let $r_{1}, r_{2}, r_{3}$, and $r_{4}$ be the four small square regions ('the $r \mathrm{~s}$ '), $s$ be the big square fusion, and $x$ the object at the center of $s$. What our theory allows us to say in this case is that $r_{1}, r_{2}, r_{3}$, $r_{4}$, and the object $x$ taken together collectively fill $s$ even if there is no region that $x$ fills by itself, and thus also no region at which $x$ is exactly located. For each $r$ among the $r$ s we can also say that $x$ and $r$ collectively fill $r$. Therefore, $x$ helps fill every subregion of $s$ and is, thus, omnipresent at $s$. Since $x$ helps fill no region that is not part of $s, x$ is also maximally omnipresent at $s$, and so it is both weakly and entirely located at $s$. Furthermore, since each of the $r$ s overlap the region at which $x$ is maximally omnipresent, it also follows that $x$ is weakly located at each of the $r$ s. However, $x$ is not entirely located at any of the $r$ s, since it is clearly not maximally omnipresent at any of them.

Therefore, it appears possible to conclude that by extending $x x @_{>} S$ with (17) and axioms 4-6 it is possible to adequately account for both Kleinschmidt's Almond in the Void

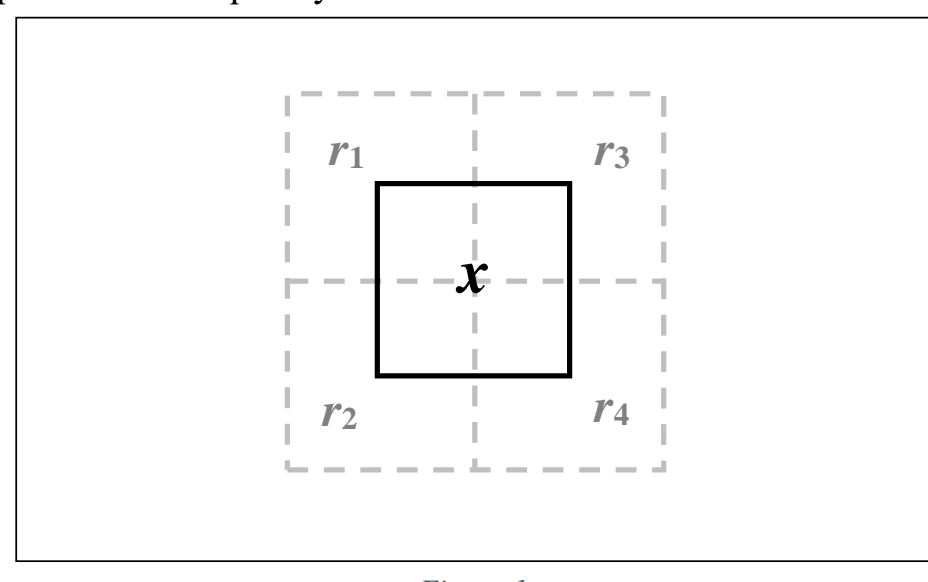

Figure 1

\footnotetext{
${ }^{18}$ See footnote 11 .
} 
and Out of Place Tiles cases. Since $x x @_{>} S$ features just one primitive notion, it follows that Almond in the Void and Out of Place Tiles are not a direct threat to parsimonious theories of location.

\section{On plural multilocation}

As it was noted above, $x x @_{>} S$ validates Functionality and, thus, makes multilocation impossible. Therefore, some may claim that the results achieved so far are at best limited, as they should be understood as conditional on the rejection of multilocation. As I will argue in this section, this objection can be resisted. ${ }^{19}$

Consider Figure 2. It represents a square object ('Bob') multilocated four times and 'covering' a circular region of space ('the circle'). Does Bob fill the circle? Well, yes and no. No, in the sense that Bob is smaller than the circle and every region at which Bob is

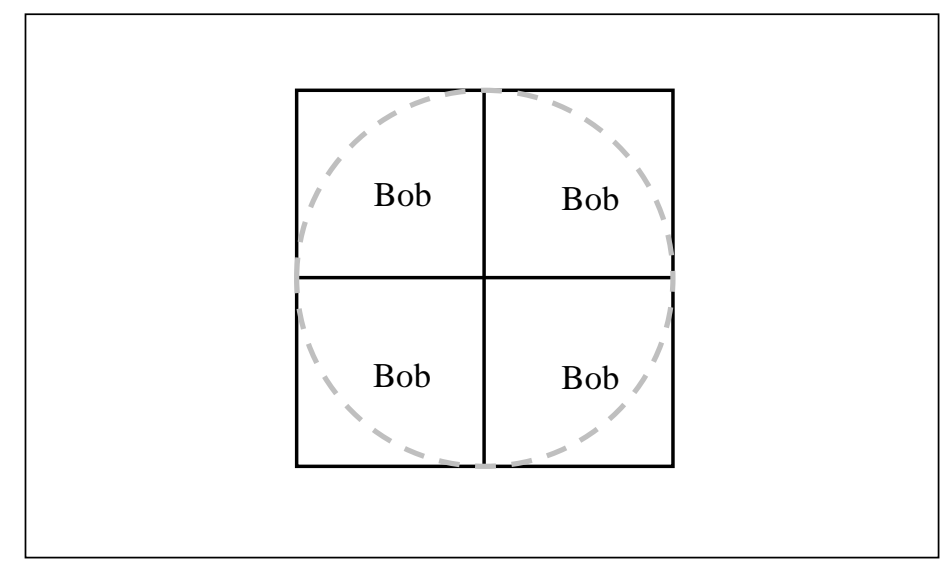

Figure 2

exactly located is smaller than the circle. Yes, in the sense that no part of the circle is 'free of Bob'. We may call these two ways of filling a certain region of space basic and derivative filling. Within a framework in which exact location is the primitive notion, basic and derivative filling are easy to define (to avoid confusion, I will use here ' $L$ ' for the relevant primitive notion of exact location and ' $L_{>}$' and ' $L_{D_{>}}$' for basic and derivative filling, respectively):

\footnotetext{
${ }^{19}$ Notice that $x x @>S$ already allows for what may be seen as a 'weak' form of multilocation. Consider, in fact, the following principle, which can be labelled 'Strong Functionality':

Strong Functionality If an object $x$ is exactly located at a region $r$, then every region $s$ at which $x$ is weakly located overlaps $r$$$
x @ r \rightarrow \forall s(x @ s \rightarrow s \circ r)
$$

Imagine that someone, mixing a gunky Spritz, spills a drop of water, so that the drop of water ends up filling by itself a region $r$ disjoint from the region of space enclosed by the gunky glass containing the gunky Spritz. In that case, $r$ is the biggest region of space that is filled by the water alone. In fact, all the other regions of space that are (partially) filled by the water are subregions of the region inside the gunky glass, but the water doesn't manage to fill any subregion of that region by itself. It follows, thus, that according to $x x S @_{>}$, the water is exactly located at $r$, even if it is also weakly located at a region that is disjoint from $r$ (that is, inside the gunky glass). However, this kind of multilocation appears to be indeed too weak to be used for the common uses of multilocation, like the locative interpretation of endurantism (see, among others: Sattig 2006, Eagle 2016, Gilmore 2018, and Leonard 2018).
} 
Basic Filling $x$ basically fills $r==_{\mathrm{df}} x$ is exactly located at a superregion of $r$ $x L_{>} r=_{d f} \exists s(x L s \wedge r \leq s)$

Derivative Filling $x$ derivatively fills $r=_{\mathrm{df}} x$ doesn't basically fill $r$ and for some plurality ss of regions $x$ is exactly located at each of the $s s$ and $r$ is part of the fusion of the $s s$

$$
x L_{D>} r=_{d f} \sim x L_{>} r \wedge \exists s s(\forall s(s \prec s s \rightarrow x L s) \wedge r \leq f(s s))
$$

However, it appears to be perfectly possible to take the notion of basic filling as primitive (for simplicity's sake in what follows I will just use 'filling' when I mean 'basic filling'). Gilmore (2018) gives the following intuitive glosses on the notion of exact and weak location:

'an entity $x$ is exactly located at a region $y$ if and only if $x$ has [...] exactly the same shape and size as $y$ and stands [...] in all the same spatial or spatiotemporal relations to other entities as does $y$.' (Gilmore, 2018: §2)

It is possible to provide a similar intuitive gloss for the primitive notion of singular basic filling:

Singular basic filling An entity $x$ basically fills a region $y$ if and only if no part of $y$ is free of $x$ and the size of $y$ is not bigger than the size of $x$

Singular basic filling is thought of as not complying with the following principle of 'Additive Filling' (for disambiguation I will use in what follows ' $\mathbb{A}_{>}$' for this primitive notion of basic filling):

Additive Filling If for some plurality ss of regions, $r$ is the fusion of the $s s$ and $x$ fills each of the $s s$, then $x$ also fills $r$ $\left(r=f(s s) \wedge \forall s\left(s \prec s s \rightarrow x \mathbb{A}_{>} s\right)\right) \rightarrow x \mathbb{A}_{>} r$

This fact allows for the possibility of multilocation if we define the notion of exact location along the lines of (11) (and, thus, as a maximal region basically filled by an entity):

$\left(11^{*}\right) x$ is exactly located at $r=\mathrm{df} x$ fills $r$ and every region that is filled by $x$ and that has $r$ as a part is identical to $r$ $x \mathbb{A} r={ }_{d f} x \mathbb{A}_{>} r \wedge \forall s\left(\left(x \mathbb{A}_{>} s \wedge r \leq s\right) \rightarrow r=s\right)^{20}$

\footnotetext{
${ }^{20}$ The notion of singular derivative filling can be defined as in Derivative Filling. Notice that, assuming axiom 3 (expressed by means of the notion of basic singular filling), this notion of derivative filling is additive in the sense of Additive Filling. Proof. Suppose that $r$ is the fusion of the $s s$ and that $x$ derivatively fills each of the ss. From the definition of derivative filling it follows that each of the $s s$ is part of a fusion of exact locations of $x$. Let $t t$ be the plurality of exact locations of $x$ that overlap some of the $s s$. It follows from classical mereology that $r$ is part of the fusion of the $t$. Axiom 3 entails that that if $x$ basically fills $r$, then it also basically fills every
} 
Consider again Figure 2. In that case, there are four square regions of space that are maximal regions basically filled by Bob, so that Bob is also exactly located at them. However, Bob doesn't basically fill the circle: it only derivatively fills it, in virtue of being exactly located at each of the four square regions.

So far, we have considered only cases of singular basic filling. We can now move on to the more general notion of plural basic filling which we can take to be our new primitive locative notion. ${ }^{21}$ An intuitive gloss of the notion of plural basic filling can be given as follows:

Plural basic filling A plurality $x x$ of entities collectively fill a region $y$ if and only if no part of $y$ is free of all the $x x$ and the size of $y$ is not bigger than the sum of the sizes of all the $x x$.

In this case, we can adopt the definitions and axioms of $x x S @_{>}$and formulate them using the notion of plural basic filling, with the only exception of Axiom 1. In fact, it is easy to check that Axiom 1 entails Additive Filling when the $x x$ are the (improper) plurality of entities that are identical to a certain entity $x$. In order to avoid this, it is sufficient to reformulate Axiom 1 by replacing the notion of 'all pervasively in' with the notion which we may label 'all uniquely in' (notice that in what follows I will take ' $\mathbb{A}_{>}$' to be multigrade in its first place): ${ }^{22}$

(21) the $x x$ are all uniquely in a plurality ss of regions $=_{\mathrm{df}}$ each of the $x x$ fills just one of the $s s$

$$
x x U_{>} s s={ }_{d f} \forall x\left(x<x x \rightarrow \exists ! s\left(s<s s \wedge x \mathbb{A}_{>} s\right)\right)
$$

Axiom 1* If for some plurality $s s$ of regions, $r$ is the fusion of the $s s$ and the $x x$ are all uniquely in the ss and pervasively cover the $s s$, then the $x x$ collectively fill $r$

$\exists s s\left(x x U_{>} s s \wedge x x \mathrm{COV}_{>} s s \wedge r=f(s s)\right) \rightarrow x x \mathbb{A}_{>} r$

Axiom $1^{*}$ doesn't entail Additive Filling. In fact, if the $x x$ are all identical to $x$ and a plurality ss of regions are covered by the $x x$, it doesn't follow that $x$ also fills their fusion $r$, as in this case the $x x$ are not all uniquely in the ss (since it is false that $x$-and, thus, each of the $x x$-fills only one of the ss). A notion of plural 'generic' filling can be defined as follows:

subregion of $r$. Each of the $s s$ is a part of $r$ that is only derivatively filled by $x$. Therefore, $x$ doesn't basically fill $r$. We have, thus, from the definition of derivative filling that $x$ derivatively fills $r$.

${ }^{21}$ Recall that the notion of plural basic filling is more general than the notion of singular basic filling because it subsumes cases of singular basic filling as cases in which the plurality of entities in question is an improper plurality, that is, a plurality of entities identical to just one entity (like the 'plurality' of things that are identical to $x$ ). See, also, footnote 8 .

${ }^{22}$ See footnote 9 . 


\section{Generic Filling}

(i) if the $x x$ fill $r$, then the $x x$ generically fill $r$; $x x \mathbb{A}_{>} r \rightarrow x x \mathbb{A}_{>}^{G} r$

(ii) if the $y y_{1}$ generically fill $s_{1}$, the $y y_{2}$ generically fill $s_{2}, \ldots$, the $y y_{n}$ generically fill $s_{n}$, the $x x$ are the plurality of entities that are identical to either one of the $y y_{1}$, one of the $y y_{2}, \ldots$, or one of the $y y_{n}$, and $r$ is the sum of the plurality of regions $\left[s_{1}, s_{2}, \ldots, s_{n}\right]$, then the $x x$ generically fill $r$; $\left(y y_{1} \mathbb{A}_{>}^{G} s_{1} \wedge y y_{2} \mathbb{A}_{>}^{G} s_{2} \wedge \ldots \wedge y y_{n} \mathbb{A} G_{>}^{G} s_{n} \wedge \forall z\left(z \prec x x \leftrightarrow\left(z \prec y y_{1} \vee z \prec\right.\right.\right.$ $\left.\left.\left.y y_{2} \vee \ldots \vee z \prec y y_{n}\right)\right) \wedge r=f\left(\left[s_{1}, s_{2}, \ldots, s_{n}\right]\right)\right) \rightarrow x x \mathbb{A}_{>}^{G} r$

(iii) in no other case the $x x$ generically fill $r$.

A plurality $x x$ of entities can then be said to derivatively fill a region $r$ if and only if, the $x x$ generically fill $r$ but they don't basically fill it.

Both the notions of singular and plural basic filling appear to be intelligible. At the same time they both appear to make multilocation possible. As for the case of multilocation involving plural basic filling, consider the possibility of an enduring gunky Spritz multilocated at two temporally disjoint regions $r$ and $s$. As in Loss's (2019) case, there is no region that either the water and the wine manage to fill by themselves. However, they collectively fill both $r$ and $s$ :

$$
(\text { wine }, \text { water }) \mathbb{A}_{>} r \wedge(\text { wine }, \text { water }) \mathbb{A}_{>} S
$$

Furthermore, we can assume that $r$ and $s$ are both maximal regions that are basically (and collectively) filled by the water and the wine taken together:

$$
\begin{aligned}
& \forall x\left(\left((\text { wine, water }) \mathbb{A}_{>} x \wedge r \leq x\right) \rightarrow r=x\right) \\
& \forall x\left(\left((\text { wine, water }) \mathbb{A}_{>} x \wedge s \leq x\right) \rightarrow s=x\right)
\end{aligned}
$$

The Spritz is here thought of as the fusion of the water and the wine, so that, intuitively, it singularly fills both $r$ and $s$ :

\section{SpritzAㄱ $>r \wedge S p r i t z \mathbb{A}_{>} S$}

Since Additive Filling is invalid in this case, there is no problem in taking $r$ and $s$ to be both maximal regions that are filled by the Spritz. It follows, therefore, the Spritz is exactly located at both $r$ and $s$, and thus, that it is multilocated:

SpritzA $r \wedge$ SpritzAs $s \wedge r \neq s$ 


\section{Conclusion}

The possibility of Nolan's (2006) Stoic Gunk and Kleinschmidt's (2016) Almond in the Void cases has been used to threaten ideologically parsimonious theories of location positing just one locative notion as primitive. As I have argued in this paper, there is no need to reject the possibility of this kind of exotica in order to keep one's theory of location ideologically tidy. In fact, a theory assuming as primitive a notion of plural pervasive location appears to be able to deal with the possibility of both Nolan's Stoic Gunk (as argued by Loss 2019) and Kleinschmidt's Almond in the Void. As I have suggested in the final part of the paper, contrary to what is claimed by Loss (2019), the impossibility of multilocation is not a price location theorists have to pay for ideological parsimony in this case, as Loss's theory $x x @_{>} S$ can be reformulated (and slightly modified) by means of a different primitive notion of plural pervasive location that seems to be not only clearly intelligible but also perfectly consistent with the possibility of multilocation. ${ }^{23}$

\section{References}

Casati, R. and Varzi, A. 1999. Parts and Places: The Structures of Spatial Representation. Cambridge, MA: MIT Press.

Eagle, A. 2016. Multiple location defended. Philosophical Studies, 173: 2215-2231.

Eagle, A. 2019. Weak Location. Dialectica 73(1-2): 149-81.

Fine, K. 2006. In Defense of Three-Dimensionalism. Journal of Philosophy 103(12): 699714

Gilmore, C. 2018. Location and Mereology. The Stanford Encyclopedia of Philosophy (Fall 2018 Edition), Edward N. Zalta, ed., URL = <http://plato.stanford.edu/archives/fall2018/entries/location-mereology/>.

Goodsell, Z., Duncan, M. and K. Miller. 2019. What is an Extended Simple Region? Philosophy and Phenomenological Research. doi:10.1111/phpr.12623.

Hudson, H. 2005. The Metaphysics of Hyperspace. Oxford University Press.

Kleinschmidt, S. 2014. Introduction. In Kleinschmidt, S. (ed.) Mereology and Location. New York: Oxford University Press: xiii-xxxiii.

Kleinschmidt, S. 2016. Placement Permissivism. Journal of Philosophy 113(3): 117-36.

Leonard, M. 2014. Locating Gunky Water and Wine. Ratio 27(3): 306-315.

Leonard, M. 2018. Enduring through gunk. Erkenntnis. 83: 753-771.

Loss, R. 2019. How to make a gunky Spritz. Thought: A Journal of Philosophy 8(4): 2509

Nolan, D. 2006. Stoic Gunk. Phronesis 51(2):162-183.

Oliver, A. and T. Smiley. 2004. Multigrade Predicates. Mind 113: 609-681.

Parsons, J. 2007. Theories of Location. In Zimmerman, D. ed., Oxford Studies in Metaphysics, Volume 3. New York: Oxford University Press: 201-32.

Sattig, T. 2006. The language and reality of time. Oxford: Oxford University Press.

Simons, P. 2014. Where It's At: Modes of Occupation and Kinds of Occupant. In Kleinschmidt, S. (ed.) Mereology and Location. New York: Oxford University Press: 59-68.

\footnotetext{
${ }^{23}$ Many thanks to two anonymous referees for this Journal.
} 